\title{
A Dilute Magnet with Ferro- and Antiferromagnetic Exchange Interactions. The Structural Fluctuations Analysis
}

\author{
R. Pikula And J. KiRkiewicz \\ Department of Physics, Maritime University \\ Wały Chrobrego 1/2, 70-500 Szczecin, Poland
}

(Received February 22, 2007; revised version January 15, 2008)

\begin{abstract}
We analysed the influence of the fluctuations in distribution of magnetic atoms on the properties of dilute magnets with mixed ferro- and antiferromagnetic exchange interactions. The high- and low concentration approximations were found to give very close results and the most important impact on the physical quantities origins from the lowest, square fluctuations which play the predominant role in the whole range of concentrations of magnetic atoms.
\end{abstract}

PACS numbers: 05.70.Jk, 75.30.Kz

\section{Introduction}

Theoretical description of dilute magnets containing magnetic and nonmagnetic atoms randomly distributed over all lattice sites is usually based on the assumption that physical parameters which describe a state of the system are the results of the averaging procedure over all possible distributions of magnetic atoms in the system. In the general case, due to their mathematical complexity the exact results are impossible to obtain, so averaging is realised in approximation. The approximation should take into account the concentration of magnetic atoms in the system and, as a consequence, the impact of the fluctuations in distribution of magnetic component on the averaged quantities. The aim of this paper is an assessment of the structural fluctuations influence on the basic parameters of the system by the use of two extreme approximations reasonable separately in the case of high and low concentrations of magnetic atoms. The calculations have been presented for the general case of the two-sub-lattice model of the site type with mixed ferro- and antiferromagnetic exchange interactions that relates to some dilute ferrimagnets with the perovskite structure such as $\mathrm{LaMn}_{1-c} \mathrm{Ni}_{c} \mathrm{O}_{3}, \mathrm{LaMn}_{1-c} \mathrm{Co}_{c} \mathrm{O}_{3}$, 
$\mathrm{La}_{1-c} \mathrm{Sr}_{c} \mathrm{CoO}_{3}$, or $\mathrm{LaMn}_{1-c} \mathrm{Ga}_{c} \mathrm{O}_{3}, \mathrm{La}_{1-c} \mathrm{X}_{c} \mathrm{MnO}_{3}$, with $\mathrm{X}=\mathrm{Ca}$, Ba, Sr. However, the derived results allow one as well to compare both the approximations in the case of dilute magnets with one kind of exchange interactions.

\section{The averaged Green function}

For a given distribution $\mathcal{R}$ of magnetic atoms, we introduce the Hamiltonian of the spin system in a form of

$$
\begin{gathered}
H(\mathcal{R})=-\frac{1}{2} \sum_{(r, s)} J_{\varepsilon}(r, s)\left[S_{r}^{z} S_{s}^{z}+\eta_{\varepsilon}\left(S_{r}^{+} S_{s}^{-}+S_{r}^{-} S_{s}^{+}\right)\right] \\
+\sum_{(r, t)} K(r, t)\left[S_{r}^{z} S_{t}^{z}+\eta_{\gamma}\left(S_{r}^{+} S_{t}^{-}+S_{r}^{-} S_{t}^{+}\right)\right]
\end{gathered}
$$

where $\sum_{(. .)}$is summing over all lattice points coupled with magnetic atoms assuming that the lattice points $r$ and $s$ belong to the same sub-lattice $\varepsilon=\alpha$ (for $r, s \in \alpha$ ) or $\varepsilon=\beta$ (for $r, s \in \beta$ ) whereas the lattice points $r$ and $t$ belong to different sub-lattices. Independently of the distribution $\mathcal{R}$, the constants $J_{\varepsilon}(r, s)$ and $K(r, t)$ denote positive integrals of exchange bonding ferromagnetically pairs of magnetic atoms inside each sub-lattice $\varepsilon$ and bonding antiferromagnetically pairs of atoms coupled with different sub-lattices, respectively. Parameters $\eta_{\varepsilon}, \eta_{\gamma} \in\langle 0,1 / 2\rangle$ determine the exchange anisotropy of the corresponding couplings.

The mathematical description of the spin system has been presented in the previous part of the work [1]. It is based on the theory of localised Green function and cumulant expansion technique used in configuration averaging procedure of the Green function over all possible magnetic atom distributions in the system. The presented calculations, as general ones, may be applied for any localised Green function whose analytical form depends on the decoupling method of a chain system of equations and the order of the equation at which the decoupling is performed. Irrespective of the type of approximation every one localised Green function $Q_{r}$ in a spectral representation $\omega$ may be presented as

$$
Q_{r}=Q_{r}\left[\omega, x_{\varepsilon 1}(r), x_{\varepsilon^{\prime} 2}(r)\right], \quad \varepsilon=\alpha, \beta, \quad \varepsilon \neq \varepsilon^{\prime} .
$$

Function $Q_{r}$ is connected with any magnetic lattice point $r$ that belongs to one of sub-lattices $\varepsilon$, but both the variables $x_{\varepsilon l}(r)$ depend only on the configuration of magnetic atoms and fluctuate around zero with the change in the configuration $\mathcal{R}$. In the approximation of the nearest neighbouring atoms the variables take a form of

$$
x_{\varepsilon l}(r)=\frac{2}{N} \sum_{\boldsymbol{k}} \frac{\gamma_{l}(\boldsymbol{k})}{c_{\varepsilon}} \exp \left(-\mathrm{i} \boldsymbol{k} \boldsymbol{R}_{r}\right) \tilde{\rho}_{\varepsilon}(\boldsymbol{k}),
$$

where summing runs over $N / 2$ vectors $\boldsymbol{k}$ of the reciprocal lattice in the first Brillouin zone. The quantity $c_{\varepsilon} \in\langle 0,1\rangle$ is concentration of magnetic atoms on sublattice $\varepsilon$ but vectors $\boldsymbol{R}_{r}$ determine the locations of magnetic lattice points $r$, at the same time for $r \in \alpha(\beta), \varepsilon \in \alpha(\beta)$ for $l=1$ or $\varepsilon \in \beta(\alpha)$ for $l=2$. Parameters 


$$
\gamma_{l}(\boldsymbol{k})=\frac{1}{z_{l}} \sum_{\boldsymbol{\delta}_{l}} \exp \left(\mathrm{i} \boldsymbol{k} \boldsymbol{\delta}_{l}\right)
$$

define the spatial crystallographic structure of the lattice described by vectors $\boldsymbol{\delta}_{l}$ that determine the locations of all $z_{1}$ inter- and $z_{2}$ intra-sub-lattice nearest neighbouring atoms. The variable on the right side of Eq. (3)

$$
\tilde{\rho}(\boldsymbol{k})=\rho_{\varepsilon}(\boldsymbol{k})-\frac{N}{2} c_{\varepsilon} \delta(\boldsymbol{k})
$$

with

$$
\delta(\boldsymbol{k})=\sum_{r \in \varepsilon} \exp \left(\mathrm{i} \boldsymbol{k} \boldsymbol{R}_{r}\right)
$$

determines fluctuation of the structural parameter

$$
\rho_{\varepsilon}(\boldsymbol{k})=\sum_{r \in \varepsilon} \xi_{r} \exp \left(\mathrm{i} \boldsymbol{k} \boldsymbol{R}_{r}\right)
$$

where summing runs over all (magnetic as well as non- magnetic) points $r$ of sublattice $\varepsilon$, but $\xi_{r}$ is zero-one operator that takes 1 with probability $c_{\varepsilon}$ when the lattice point $r$ is coupled with a magnetic atom and takes 0 with probability $1-c_{\varepsilon}$ in the other case. Expanding the Green function (2) into Taylor's series in relation to the variables $x_{\varepsilon l}(r)$ and averaging the obtained result over all possible configurations of magnetic atoms in the system, we find

$$
\begin{gathered}
Q_{\varepsilon}(\omega) \delta(\boldsymbol{k})=\left[D_{r}^{(2)} Q_{r}\right]_{0}\left(\frac{2}{N}\right)^{\nu} \sum_{\left\{\boldsymbol{K}_{\}}\right.} \delta\left(\boldsymbol{k}-\sum_{l, f} \boldsymbol{k}_{l f}\right) \\
\prod_{l=1}^{2}\left\langle\left\langle\prod_{f=1}^{\nu_{l}} \tilde{\rho}_{\varepsilon}\left(\boldsymbol{k}_{l f}\right)\right\rangle\right\rangle \prod_{f=1}^{\nu_{l}} \frac{\gamma_{l}\left(\boldsymbol{k}_{l f}\right)}{c_{\varepsilon}},
\end{gathered}
$$

where

$$
\begin{aligned}
& {\left[D_{r}^{(2)} Q_{r}\right]_{0}=\sum_{\nu=0}^{\infty} \frac{1}{\nu !} \sum_{\nu_{1}+\nu_{2}=\nu} \frac{\nu !}{\nu_{1} ! \nu_{2} !}\left[\frac{\partial^{\nu} Q_{r}\left(\omega, x_{\varepsilon 1}, x_{\varepsilon^{\prime} 2}\right)}{\partial x_{\varepsilon 1}^{\nu_{1}} \partial x_{\varepsilon^{\prime} 2}^{\nu_{2}}}\right]_{0},} \\
& \{\boldsymbol{K}\}=\left\{\boldsymbol{k}_{11}, \boldsymbol{k}_{12}, \ldots, \boldsymbol{k}_{1 \nu_{1}}, \boldsymbol{k}_{21}, \boldsymbol{k}_{22}, \ldots, \boldsymbol{k}_{2 \nu_{2}}\right\} .
\end{aligned}
$$

In the above equation, $\langle\langle\ldots\rangle\rangle$ denotes an average over all possible configurations of magnetic atoms in the system but summing in Eq. (9) runs over all combinations $\nu_{1}$ and $\nu_{2}$ so that $\nu_{1}+\nu_{2}=\nu \in\langle 0, \infty)$. Using the basic rules of the cumulant expansion technique [2] the configuration moments on the right side of Eq. (8) may be presented in a form of the sum over all possible products of the cumulants

$$
\left\langle\left\langle\prod_{\{f\}} \rho_{\varepsilon}\left(\boldsymbol{k}_{l f}\right)\right\rangle\right\rangle_{c}=\frac{N}{2} P_{n}\left(c_{\varepsilon}\right) \delta\left(\sum_{\{f\}} \boldsymbol{k}_{l f}\right),
$$

where $n$ is the order of the cumulant equal to the number of terms $\rho_{\varepsilon}\left(\boldsymbol{k}_{l f}\right),\{f\}$ - each possible subset of numbers from 1 to $\nu_{l}$, but $P_{n}\left(c_{\varepsilon}\right)$ is a polynomial of $n$-order in relation to $c_{\varepsilon}$ written in 


$$
P_{n}\left(c_{\varepsilon}\right)=\left[\frac{\mathrm{d}^{n}}{\mathrm{~d} \lambda^{n}} \ln \left(1-c_{\varepsilon}+c_{\varepsilon} \mathrm{e}^{\lambda}\right)\right]_{\lambda=0} .
$$

Taking into account the foregoing notations, the first terms of the averaged Green function (8) may be expressed in the algebraic expansion

$$
\begin{aligned}
Q_{\varepsilon}(\omega) & -Q_{\varepsilon}^{0}(\omega)=\frac{1}{2 !} \sum_{l=1}^{2}\left(\frac{\partial^{2} Q_{r}}{\partial x_{\varepsilon l}^{2}}\right)_{0}\left[\frac{P_{2}\left(c_{\varepsilon}\right)}{c_{\varepsilon}^{2}} \frac{2}{N} \sum_{\boldsymbol{k}_{l 1}} \gamma_{l}^{2}\left(\boldsymbol{k}_{l 1}\right)\right] \\
+ & \frac{1}{3 !} \sum_{l=1}^{2}\left(\frac{\partial^{3} Q_{r}}{\partial x_{\varepsilon l}^{3}}\right)_{0}\left[\frac{P_{3}\left(c_{\varepsilon}\right)}{c_{\varepsilon}^{3}}\left(\frac{2}{N}\right)^{2} \sum_{\boldsymbol{k}_{l 1}} \sum_{\boldsymbol{k}_{l 2}} \gamma_{l}\left(\boldsymbol{k}_{l 1}\right) \gamma_{l}\left(\boldsymbol{k}_{l 2}\right) \gamma_{l}\left(\boldsymbol{k}_{l 1}+\boldsymbol{k}_{l 2}\right)\right] \\
+ & \frac{1}{4 !} \sum_{l=1}^{2}\left(\frac{\partial^{4} Q_{r}}{\partial x_{\varepsilon l}^{4}}\right)_{0}\left\{\frac { P _ { 4 } ( c _ { \varepsilon } ) } { c _ { \varepsilon } ^ { 4 } } ( \frac { 2 } { N } ) ^ { 3 } \sum _ { \boldsymbol { k } _ { l 1 } } \sum _ { \boldsymbol { k } _ { l 2 } } \sum _ { \boldsymbol { k } _ { l 3 } } \left[\gamma_{l}\left(\boldsymbol{k}_{l 1}\right) \gamma_{l}\left(\boldsymbol{k}_{l 2}\right)\right.\right. \\
& \left.\left.\times \gamma_{l}\left(\boldsymbol{k}_{l 3}\right) \gamma_{l}\left(\boldsymbol{k}_{l 1}+\boldsymbol{k}_{l 2}+\boldsymbol{k}_{l 3}\right)\right]+3\left[\frac{P_{2}\left(c_{\varepsilon}\right)}{c_{\varepsilon}^{2}} \frac{2}{N} \sum_{\boldsymbol{k}_{l 1}} \gamma_{l}^{2}\left(\boldsymbol{k}_{l 1}\right)\right]^{2}\right\} \\
+ & \frac{1}{2 ! 2 !}\left(\frac{\partial^{4} Q_{r}}{\partial x_{\varepsilon 1}^{2} \partial x_{\varepsilon^{\prime} 2}^{2}}\right)_{0} \prod_{l=1}^{2}\left[\frac{P_{2}\left(c_{\varepsilon}\right)}{c_{\varepsilon}^{2}} \frac{2}{N} \sum_{\boldsymbol{k}_{l 1}} \gamma_{l}^{2}\left(\boldsymbol{k}_{l 1}\right)\right]+\ldots
\end{aligned}
$$

where

$$
Q_{\varepsilon}^{0}(\omega)=Q_{r}\left[\omega, x_{\varepsilon 1}(r)=0, x_{\varepsilon^{\prime} 2}(r)=0\right]
$$

is the Green function obtained as a result of neglecting the fluctuations in the distribution of magnetic atoms in the system. The right side of Eq. (13) is then a contribution to the averaged Green function that origins from all structural fluctuations.

Using the rules for calculation of cumulants we may show that for $\nu=5$ the series (13) expands by 24 additional elements which reduce to 6 elements after summing of the algebraically even terms. For $\nu=6$ the number of elements increases by 61 , giving additional 11 uneven terms of the expansion. Hence, when the quantity $\nu$ increases, as it is connected with higher correlations of structural fluctuations, the interpretation of the impact of each term of the expansion (13) on the Green function becomes a problem. However, to assess the impact of structural fluctuations on the averaged Green function it is essential to take into account the terms with the high values of $\nu$. It results from the fact that at the decoupling of the moments of even very high orders into cumulants, in the series there are the terms which at the given approximation may substantially influence the averaged Green function in the way as the terms that origin from the moments of the low orders do. For example, expanding the Green function into series and taking into account only linear dependence of its terms on one concentration $c_{\varepsilon}$, all elements of the expansion including for given $\nu$ only polynomials $P_{\nu}\left(c_{\varepsilon}\right)$, which for low $c_{\varepsilon}$ are proportional to $c_{\varepsilon}$, should be considered. But expanding the Green 
function in relation to $c_{\varepsilon}^{2}$, the terms including either $P_{\nu}\left(c_{\varepsilon}\right)$ or $P_{\nu-r}\left(c_{\varepsilon}\right) P_{r}\left(c_{\varepsilon}\right) \sim c_{\varepsilon}^{2}$ should be considered as well as all the other terms should be excluded. In both approximations the terms which should be taken into account appear in the terms in which $\nu$ has low and a high value as well.

The discussion on structural fluctuations connecting with moments of high orders is also essential in other methods of calculations of averaged quantities. In the literature the methods of selective summing of a series are quite often used approximations. Such approaches consider only these elements in the terms of the series which are responsible for the impact that origins from the structural fluctuations of the given order. In such formulated approximation the expansion of moments into cumulants even for high $\nu$ gives the terms whose impact on the averaged Green function is physically equivalent to the influence of terms connected with moments of lower orders. For example, in the moment of the fourth order which appears in Eq. (13) the terms with binary interpretation of structural correlation occur.

\section{Quantitative analysis}

The quantitative analysis was carried out for critical concentration that means such the lowest concentration of magnetic atoms below which the longrange order declines. The assessment refers to the approximation of the effective field that is a result of a given scheme of self-consistency of the chain system of equations for the Green function introduced for solid magnets by Riess and Movroyannis [3, 4]. Carrying out the calculations quite similar to those performed in the previous paper [1] we find one-particle Green function, which for the spectral representation takes the form of

$$
G_{r}\left(\omega, x_{\varepsilon 1}, x_{\varepsilon^{\prime} 2}\right)=\frac{\omega+\Gamma_{r}\left(x_{\varepsilon 1}, x_{\varepsilon^{\prime} 2}\right)}{\left[\omega+\Delta_{r}\left(x_{\varepsilon 1}, x_{\varepsilon^{\prime} 2}\right)\right]\left[\omega-\Delta_{r}\left(x_{\varepsilon 1}, x_{\varepsilon^{\prime} 2}\right)\right]},
$$

where

$$
\begin{aligned}
& \Delta_{r}\left(x_{\varepsilon 1}, x_{\varepsilon^{\prime} 2}\right)=\left[\Gamma_{r}^{2}\left(x_{\varepsilon 1}, x_{\varepsilon^{\prime} 2}\right)+\Omega_{r}^{2}\left(x_{\varepsilon 1}, x_{\varepsilon^{\prime} 2}\right)\right]^{1 / 2}, \\
& \Gamma_{r}\left(x_{\varepsilon 1}, x_{\varepsilon^{\prime} 2}\right)=J_{\varepsilon}\left\langle S_{\varepsilon}^{z}\right\rangle z_{1} c_{\varepsilon}\left(1+x_{\varepsilon 1}\right)-K\left\langle S_{\varepsilon^{\prime}}^{z}\right\rangle z_{2} c_{\varepsilon^{\prime}}\left(1+x_{\varepsilon^{\prime} 2}\right), \\
& \varepsilon=\alpha, \beta, \quad \varepsilon \neq \varepsilon^{\prime}, \\
& \Omega_{r}\left(x_{\varepsilon 1}, x_{\varepsilon^{\prime} 2}\right)=\left[J_{\varepsilon}^{2}\left(\frac{1}{4}+\eta_{\varepsilon}^{2}-\left\langle S_{\varepsilon}^{z}\right\rangle^{2}\right) z_{1} c_{\varepsilon}\left(1+x_{\varepsilon 1}\right)\right. \\
& \left.+K^{2}\left(\frac{1}{4}+\eta_{\gamma}^{2}-\left\langle S_{\varepsilon^{\prime}}^{z}\right\rangle^{2}\right) z_{2} c_{\varepsilon^{\prime}}\left(1+x_{\varepsilon^{\prime} 2}\right)\right]^{1 / 2} .
\end{aligned}
$$

Above, the site-dependent variables $x_{\varepsilon l}(r)$ are given by Eq. (3) but $J_{\varepsilon}$ and $K$ denote positive ferro- and antiferromagnetic exchange constants reduced to the nearest neighbours. In order to find the configurationally averaged Green function we expand it in a power series of $x_{\varepsilon l}(r)$ and then using the cumulant technique we express it in a form of Eq. (13). The averaged Green function with the known spectral relations determines the implicit and also expressed in the series form 
equations for the expectation values $\left\langle S_{\varepsilon}^{z}\right\rangle$ and therefrom the magnetization and the critical temperature $T_{N}$ of the system. In the limit of $T_{N} \rightarrow 0$ we find the equation for the critical concentration which for the case of $J_{\alpha}=J_{\beta} \equiv J$ and $c_{\alpha}=c_{\beta} \equiv c$ takes the form

$$
\begin{aligned}
& \varphi_{n}(c) \equiv \sum_{\nu=0}^{n} \frac{1}{\nu !} \sum_{\nu_{1}+\nu_{2}=\nu} \frac{\nu !}{\nu_{1} ! \nu_{2} !}\left[\frac{\partial^{\nu} F\left(c, x_{1}, x_{2}\right)}{\partial x_{1}^{\nu_{1}} \partial x_{2}^{\nu_{2}}}\right]_{x_{1}, x_{2}=0} \\
& \times\left\langle\left\langle x_{1}^{\nu_{1}}(r)\right\rangle\right\rangle\left\langle\left\langle x_{2}^{\nu_{2}}(s)\right\rangle\right\rangle=0, \\
& F\left(c, x_{1}, x_{2}\right)=\left(\frac{c}{1+4 \eta^{2}}\right)^{\frac{1}{2}} \frac{z_{1}\left(1+x_{1}\right)+z_{2} \kappa\left(1+x_{2}\right)}{\left[z_{1}\left(1+x_{1}\right)+z_{2} \kappa^{2}\left(1+x_{2}\right)\right]^{\frac{1}{2}}}-1,
\end{aligned}
$$

where $\kappa=K / J$ but $\eta_{\varepsilon}=\eta_{\gamma} \equiv \eta \in\langle 0,1 / 2\rangle$ determines exchange anisotropy of the interactions. The parameters $x_{l}(r)$, defined for $c_{\alpha}=c_{\beta}$ by Eq. (3), fluctuate around zero with the change in configurations of atoms in the nearest neighbourhood of $r$-magnetic point. For $n \rightarrow \infty$, the equation shows the accurate relation for the critical concentration of the system and it may be used to compare the results obtained from the approximations calculated for high and low magnetic atoms concentrations separately. In order to sum the series (19) the approximation which concerns the impact of the lowest, square structural fluctuations is recommended [5-7]:

$$
\left\langle\left\langle x_{l}^{\nu_{l}}(r)\right\rangle\right\rangle_{\mathrm{HC}}= \begin{cases}\left\langle\left\langle x_{l}^{2}(r)\right\rangle\right\rangle^{\frac{\nu_{l}}{2}}=\left[\frac{1}{z_{l} c^{2}} P_{2}(c)\right]^{\frac{\nu_{l}}{2}} & \text { for } \nu_{l} \text { even, } \\ 0 & \text { for } \nu_{l} \text { uneven. }\end{cases}
$$

This approximation is strictly fulfilled within the limits of high magnetic atoms concentrations for which structural fluctuations of the lowest orders are significantly more probable than the structural fluctuations of high orders [8]. For low concentrations of magnetic atoms the approximation concerning the terms including the first power $c_{\varepsilon}$ in the expansion (19) is sensible [2, 9]:

$$
\left\langle\left\langle x_{l}^{\nu_{l}}(r)\right\rangle\right\rangle_{\mathrm{LC}}=\frac{1}{z_{l}^{\nu_{l}-1} c^{\nu_{l}}} P_{\nu_{l}}(c) .
$$

In Table there are presented the critical concentrations $c_{n}$ in the approximation of the low and high concentrations which are for $n=2, \ldots, 6,10$ the solutions of equations $\varphi_{2}(c)=1, \ldots, \varphi_{6}(c)=1, \varphi_{10}(c)=1$, respectively. The critical concentrations

$$
c_{1}=\frac{\left(1+4 \eta^{2}\right)\left(z_{1}+\kappa^{2} z_{2}\right)}{\left(z_{1}+\kappa z_{2}\right)^{2}}
$$

are the solutions of Eq. (19) obtained when neglecting the fluctuations in the magnetic atoms distribution. The accurate values of the critical concentrations in both approximations are the limits of sequence of solutions $c_{n}$ for $n \rightarrow \infty$. In the approximation of high concentrations the limit of the sequence is determined by the equation 
TABLE

The critical concentrations $c_{n}$ obtained in the approximation of low concentrations (LC) and high concentrations (HC) of magnetic atoms for $\kappa=0.1$ and various pairs $\left(z_{1}, z_{2}\right)$ of co-ordinate numbers for the Heisenberg model $(\eta=1 / 2)$. For comparison, the results for dilute magnets with one kind of exchange interaction and various coordinate numbers $z$ were presented. The accuracy of the calculations $\Delta c_{n}= \pm 0.0001$.

\begin{tabular}{c|c|c|c|c|c|c|c||c|c}
\hline \hline$\left(z_{1}, z_{2}\right)$ & $c_{n}$ & $c_{1}$ & $c_{2}$ & $c_{3}$ & $c_{4}$ & $c_{5}$ & $c_{6}$ & $c_{10}$ & $c_{\infty}$ \\
$z$ & & & & & & & & & \\
\hline$(4,2)$ & LC & 0.4558 & 0.4820 & 0.4818 & 0.4810 & 0.4810 & 0.4812 & 0.4811 & \\
& HC & 0.4558 & 0.4820 & 0.4820 & 0.4837 & 0.4837 & 0.4838 & 0.4839 & 0.4839 \\
$(6,2)$ & LC & 0.3132 & 0.3371 & 0.3353 & 0.3348 & 0.3351 & 0.3351 & 0.3351 & \\
& HC & 0.3132 & 0.3371 & 0.3371 & 0.3391 & 0.3391 & 0.3393 & 0.3394 & 0.3394 \\
$(6,6)$ & LC & 0.2782 & 0.2973 & 0.2957 & 0.2956 & 0.2957 & 0.2957 & 0.2958 & \\
& HC & 0.2782 & 0.2973 & 0.2973 & 0.2985 & 0.2985 & 0.2986 & 0.2985 & 0.2985 \\
$4(\mathrm{D})$ & LC & 0.5000 & 0.5290 & 0.5294 & 0.5285 & 0.5284 & 0.5286 & 0.5286 & \\
& HC & 0.5000 & 0.5290 & 0.5290 & 0.5309 & 0.5309 & 0.5310 & 0.5311 & 0.5311 \\
6 & LC & 0.3333 & 0.3595 & 0.3579 & 0.3572 & 0.3575 & 0.3576 & 0.3575 & \\
$(\mathrm{SC})$ & HC & 0.3333 & 0.3595 & 0.3595 & 0.3618 & 0.3618 & 0.3621 & 0.3622 & 0.3622 \\
8 & LC & 0.2500 & 0.2723 & 0.2700 & 0.2697 & 0.2700 & 0.2700 & 0.2699 & \\
$(\mathrm{BCC})$ & HC & 0.2500 & 0.2723 & 0.2723 & 0.2744 & 0.2744 & 0.2748 & 0.2749 & 0.2749 \\
12 & LC & 0.1667 & 0.1833 & 0.1809 & 0.1811 & 0.1812 & 0.1811 & 0.1811 & \\
$($ FCC $)$ & HC & 0.1667 & 0.1833 & 0.1833 & 0.1851 & 0.1851 & 0.1854 & 0.1855 & 0.1856 \\
\end{tabular}

$$
\begin{aligned}
& \frac{1}{4}\left(\frac{c}{1+4 \eta^{2}}\right)^{\frac{1}{2}} \sum_{i, j= \pm 1} \frac{z_{1}\left(1+\mathrm{i} \xi_{1}\right)+\kappa z_{2}\left(1+j \xi_{2}\right)}{\left[z_{1}\left(1+i \xi_{1}\right)+\kappa^{2} z_{2}\left(1+j \xi_{2}\right)\right]^{\frac{1}{2}}}=1, \\
& \xi_{l}=\left[\frac{P_{2}(c)}{z_{l} c^{2}}\right]^{\frac{1}{2}}, \quad l=1 \text { or } 2,
\end{aligned}
$$

whose solutions for the Heisenberg model $(\eta=1 / 2)$ and the lattices with $\left(z_{1}, z_{2}\right)=(4,2),(6,2),(6,6)$ are the corresponding critical concentrations $c_{\infty}=$ $0.4839,0.3394,0.2985$. By comparison between the foregoing solutions and corresponding quantities $c_{n}$ plotted in Table we find that at the accuracy of the calculations $( \pm 0.0001)$, Eq. (19) determines the accurate values of the critical concentrations even for $n=6$. In the approximation of low concentrations, the extrapolated values of the critical concentrations for the Heisenberg model and $\left(z_{1}, z_{2}\right)=(4,2),(6,2),(6,6)$ are, respectively, $0.4811,0.3351,0.2958$ and are lower than the corresponding values obtained from the approximation of the high concentrations round about of $0.6,1.3,0.9 \%$. Such small differences between the results obtained from two extreme approximations may be explained by the fact that in one as well as in the other case the most substantial number contributions to the critical concentrations origin from the square structural fluctuations which 
appear at the second step of the approximations. The same critical concentrations $c_{2}$ calculated from the $\varphi_{2}(c)=1$ equation correspond to the approximations. For example, for $\left(z_{1}, z_{2}\right)=(6,2)$, in both the cases $c_{2}$ is higher than $c_{1}$ round about of $7.6 \%$ but numerical corrections that should be taken into account in $c_{2}$ to obtain the accurate values of the critical concentrations in the approximation of low and high concentrations are, correspondingly, 0.5 and $0.7 \%$ of $c_{2}$. This result is an evidence of a significant impact of the lowest structural fluctuations on the numerical results obtained also within the limits of low concentrations of magnetic atoms. It is worth noticing that in the approximation (21) the correlations of structural fluctuations of uneven orders are neglected. In the discussed model the distribution of magnetic and non-magnetic atoms on each sub-lattice is binomial and then it is symmetrical for concentrations $c_{\varepsilon}=1 / 2$ of every magnetic component. Hence, the approximation (21) is also sensible for intermediate ranges of magnetic atoms concentrations in a system.

\section{Conclusions}

We estimated the structural fluctuations impact on the critical concentration of dilute spin system with two kinds of exchange interactions. The quantitative assessment was carried out by the use of two approximations separately sensible in the limit of high and low concentrations of magnetic atoms. The numerical results obtained for all the discussed lattices are very close and they did not distinguish in both the approximations of more than 1.3\%. Such small differences are explained by the fact that the greatest contribution to the physical quantities comes from the lowest, square structural fluctuations which are the same at the second step for both approximations whereas all contributions coming from all fluctuations of the higher order lead only to correction of one order lower quantity.

\section{References}

[1] R. Pikuła, J. Kirkiewicz, Acta. Phys. Pol. A 110, 25 (2006).

[2] F. Yonezawa, T. Matsubara, Prog. Theor. Phys. 35, 357, 759 (1966).

[3] I. Riess, Physica 74, 496 (1974).

[4] I. Riess, C. Movroyannis, Physica 75, 483 (1974).

[5] T. Kaneyoshi, Prog. Theor. Phys. 44, 328 (1970).

[6] T. Kaneyoshi, R. Honmura, J. Phys. C 5, L65 (1972).

[7] T. Kaneyoshi, J. Phys. C 5, L107 (1972).

[8] A.B. Harris, J. Phys. C 7, 1671 (1974).

[9] K. Yamamoto, T. Kaneyoshi, Prog. Theor. Phys. 50, 1416 (1973). 